\title{
The Application of Magnetic Resonance in Spinal Cord Disorders
}

\author{
M. Perovitch, M.D. \\ Professor, Department of Radiology, The University of Connecticut School of \\ Medicine and Health Center, Farmington, Connecticut 06032, U.S.A.
}

\begin{abstract}
Summary
The introduction of the proton magnetic resonance imaging into clinical practice shows significant diagnostic potentials, and throws a new light on pathological changes involving the spinal cord, in particular on those related to trauma and its sequelae. The initial experience concerning the use of the proton magnetic resonance imaging in 28 patients, in whom paraplegia or quadriplegia developed following an injury to the spine, indicates that magnetic resonance imaging has specific advantages over other investigative modalities. Magnetic resonance imaging techniques permit, in most instances, a distinct demarcation of the spinal cord in its entire length or in segments, without ionising radiation or intrathecal introduction of contrast media. A significant superiority of magnetic resonance imaging is the feasibility to apply multiplanar imaging of the spinal cord without moving the paralysed patient and the possibility to repeat the imaging, even on an outpatient basis, without a major discomfort to the patient. Most of the post-traumatic lesions can be identified in magnetic resonance images, however, the application of paramagnetic enhancers may have to be considered in order to achieve a distinct delineation of a lesion from the surrounding normal spinal cord tissue in selected cases. Despite some current limitations, magnetic resonance imaging has opened new avenues to obtain information about the anatomy, and, in particular, the biochemistry of the spinal cord.
\end{abstract}

Key words: Magnetic resonance imaging; Spinal cord injury and sequelae.

\section{Introduction}

Magnetic resonance imaging (MRI) permits new information about the anatomy and, in particular, the biochemistry of the spinal cord. The notable advancements in the clinical application of magnetic resonance (MR) spinal cord imaging, achieved mainly in the course of the past year, are presented in Table I.

With MR the spinal axis can be imaged simultaneously in multiple planes without ionising radiation or intrathecal administration of contrast agents. A further significant superiority of MR is the possibility to repeat the imaging on an outpatient basis, without major discomfort to the patient. This is especially 
Table I Advantages of MRI of the spinal cord

1. No ionising radiation;

2. Feasibility of multiplanar imaging without bone artifacts;

3. Visualization of the spinal cord without intrathecal introduction of contrast media;

4. MRI can be performed on an outpatient basis;

5. MRI can be repeated without known biological hazards and major discomfort to the patient.

Table II Main physical characteristics that MR can measure

1. $T_{1}$-longitudinal relaxation (inversion-recovery, IR);

2. $\mathrm{T}_{2}$-transverse relaxation (spin-echo, $\mathrm{SE}$ );

3. Chemical shift;

4. Concentration of nuclei (hydrogen, phosphorus, sodium, etc.);

5. Volume flow.

significant for paralysed patients who may need a relatively frequent follow-up upon surgical or radiation treatment.

Magnetic resonance measures specific parameters which we presented in summary in Table II. Its functioning is based on magnetic and radio frequency fields. Thus its physical foundation differs from that of other neuroradiological modalities which have been utilising a specific type of ionising radiation in order to produce images of the morphological state of the spinal cord. Hence, the physical properties of computed tomography, angiography, or myelography are not comparable with those of magnetic resonance (Perovitch, 1985).

Presently, the most commonly used nucleus for MRI of the spinal cord is that of hydrogen, a single proton $\left({ }^{1} \mathrm{H}\right)$, which proved to be very susceptible to the influence of the magnetic field and, in addition, is found in profusion in its chemical bound forms such as water or lipids (Perovitch, 1985).

Although it is not within the scope of this presentation to analyse in detail MRI techniques, it should be mentioned that the selection of appropriate pulse sequence techniques in the clinical MRI of the spinal cord is decisive, because the appearance of both normal and abnormal structures varies very much with the imaging sequence used. Applying short echo-time (TE) and repetitiontime (TR) the contrast between the spinal cord and the cerebrospinal fluid will be maximised in spin-echo (SE) images (Fig. 1). However, there is no clear separation between the subarachnoid space and extradural tissues such as the ligaments, except for the epidural fat that appears brighter. Due to this lack of separation between the intra- and extrathecal structures the size of the subarachnoid space may be overestimated in MR images. If this separation is, however, needed, longer echo-time and repetition-time are used. In this way a delineation of the extradural components will be achieved, but the definition between the spinal cord and the cerebrospinal fluid will be lost. These are the essential characteristics of MRI, which determine the quality of spinal cord images (Han et al., 1984).

Sagittal MR images provide visualisation of the spinal cord in its entire length or in segments if a detailed analysis is needed. In Figure 1 the cervicomedullary junction and its longitudinal relationship with the structures of the posterior fossa are clearly seen without the interference of bone artifacts. 


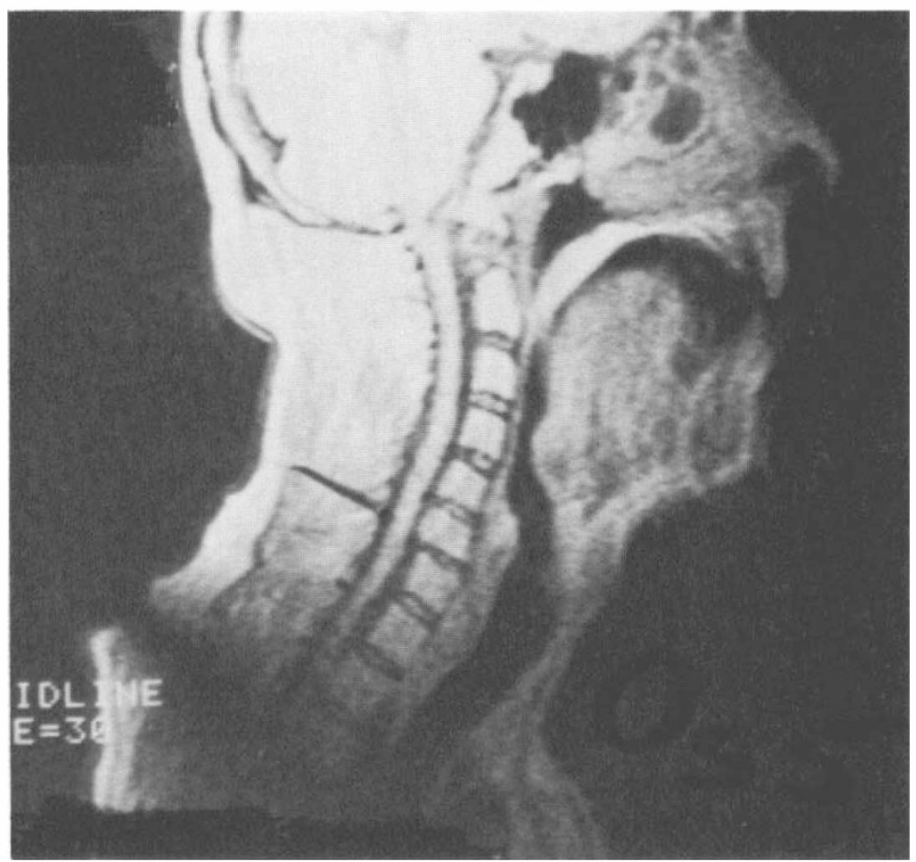

Figure 1 Applying short echo-time and repetition-time the contrast between the spinal cord and the cerebrospinal fluid is maximised in this MR image of the cervical area. Although the patient had a motor vehicle accident associated with an injury to the cervical spine, there is no evidence in this MR image of spinal cord involvement.

Different segments of the spinal cord can appear magnified on multiplanar MR images, offering further possibilities for the study of subtle changes in and around the spinal cord (Fig. 2). It should be emphasised that multiplanar MR images can be obtained without changing the position of the patient.

At this stage in our experience with the MRI of the spinal cord, some difficulties may occur in the thoracic area, and the dorsal segment of the spinal cord is not always clearly visualised. This may be due to the constant motion of the thorax, but also to our limited experience with regard to the optimal imaging techniques (Paushter et al., 1984).

On coronal images, the normal curvatures of the spine does not permit the delineation of the spinal cord in its entire length. Consequently scanning of multiple levels is required in order to examine the spinal cord in this projection.

The conus medullaris is usually seen distinctly in sagittal and coronal images, but the filum terminale and nerve fibres, however, cannot be identified. Also, the individual nerve roots exiting through the neural foramina along the spine are seldom recognisable.

\section{Results}

In the course of the last year the clinical efficacy and accuracy of MRI was analysed in 28 patients whose images showed either acute or permanent posttraumatic changes of the spinal cord. The experience with the application of 


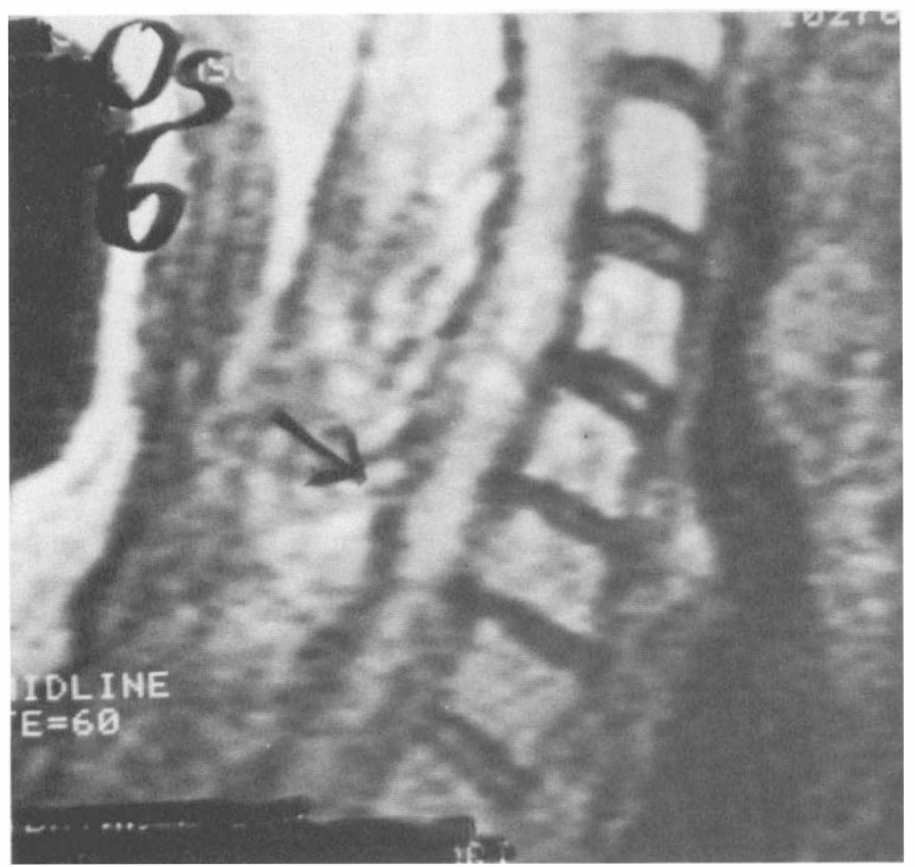

Figure 2 This is a magnified image of the cervical spinal cord. The magnification technique of multiplanar MR images offers further possibilities for the study of subtle changes in and around the spinal cord.

MRI in spinal cord injuries is still limited, and further observations are needed to broaden our present knowledge concerning the meaningful use of this diagnostic modality and interpretation of images of the traumatised spinal cord (Perovitch, 1986, I).

The MRI of the thoraco-cervical spine clearly showed the presence and extent of epidural hematoma associated with spinal cord compression in three patients, following spinal trauma in motor vehicle accidents. The post-traumatic evaluation of the spinal cord can be achieved at once in the acute phase by means of MRI without major discomfort to the patient or the risk of worsening the patient's condition. Due to these features MRI appears to be the optimal modality for the visualisation of the spinal cord under emergency conditions, and it has been replacing other diagnostic techniques, specifically myelography (Perovitch, 1981).

In four patients, in an acute stage of spinal trauma related to different motor vehicle accidents, MRI distinctly delineated spinal cord enlargement, with the central low signal intensity of intramedullary haemorrhage thus offering precise data for consideration of possible surgical treatment.

In the next three patients the sagittal $M R$ images of the cervical region revealed fractures, with or without dislocation of the bony fragments, of the vertebrae at different levels (Fig. 3). The ventral and dorsal subarachnoid compartments appeared to be open, without indications of spinal cord compression, although the initial plegia, that occurred as a consequence of automobile acci- 


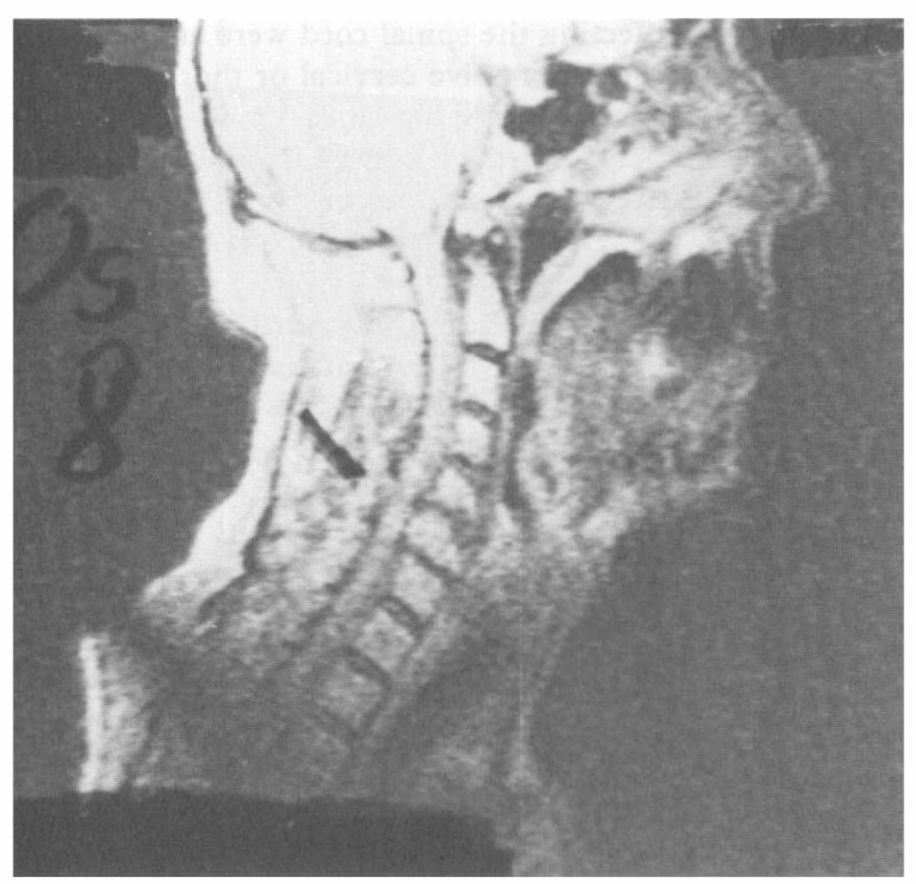

Figure 3 Following an injury to the cervical vertebrae the MR image does not demonstrate spinal cord compression.

dents, remained unchanged. In these situations MRI did not provide information about the state of the spinal cord.

In a further five patients the MR images disclosed a post-traumatic cervical and thoracic syrinx, at the preoperative stage. The selection of appropriate imaging techniques becomes of primary importance in order to display the lowsignal intensity intramedullary cavity, whereas the enlargement or external distortion of the spinal cord can be readily appreciated. The evaluation of the nature of the spinal cord enlargement may be difficult, because various other pathological processes can create a similar picture. Nevertheless, the actual experience with MRI as the investigative method for syringohydromyelia is promising, because it has the potentials to provide data not attainable by means of other diagnostic techniques (Han et al., 1984; Perovitch, 1981).

MR images following the surgical decompression were analysed in three of the above mentioned five cases. The reduction in size of the spinal cord was evident, although it appeared to be affixed to the posterior thecal wall, most likely due to the postsurgical adhesions.

Moreover, post-traumatic hydromyelia in three paraplegic patients showed clearly demarcated swelling of the spinal cord in MR images. One was seen in the lower thoracic, and the other two in the upper thoracic spinal cord. The MR characteristics of hydromyelia are difficult to discern from those seen in syringomyelia. The term hydromyelia is used here only on the basis of surgical and histological examination (Perovitch, 1981). 
Postoperative changes affecting the spinal cord were analysed in MR images in five patients. All had a decompressive cervical or thoracic surgery for acute extensive post-traumatic, epidural or intramedullary haemorrhage. In these cases varying degrees of atrophy of the spinal cord was noticable in MR images as well as deformities and a fixed change of the cord's position. The subarachnoid compartments, either the anterior or the posterior, or both, were considerably enlarged.

Finally, one should emphasise that the spinal cord in MR images lends itself to accurate measurements similar to those that can be obtained in gasmyelograms. Diffuse or more restricted post-traumatic cervico-thoracic spinal cord atrophy was analysed in five additional cases that were not operated upon. The diameters of the spinal cord can be measured in multiplanar projections without moving the patients (Perovitch, 1983; Perovitch, 1981).

\section{Discussion}

Although clinical experience is so far limited, it is reasonabie, at this stage, to consider MRI as a valuable diagnostic tool for the evaluation of an injured spinal cord. Some of its characteristic features mentioned in Table I render this diagnostic modality especially attractive for the use in shock-trauma services where a prompt assessment of the spinal cord state is essential for possible eventual surgical treatment, and it also proved to be useful in the follow-up of paraplegic patients (Perovitch, 1981).

Although the external appearance of the spinal cord and its surroundings can be estimated in most cases in MR images, the internal post-traumatic changes, such as a cyst formation, cannot always be recognised. Thus concerning the feasibility to determine the probable nature of some lesions of the spinal cord, MRI has not yet yielded satisfactory results. In such situations it is necessary to combine MRI with other current diagnostic modalities, especially computed tomography or spinal cord angiography. The next, recent, possibility would be to enhance MR images with a paramagnetic enhancer.

Initially, it was assumed that endogenous variations in relaxation times could provide a subtle distinction among anatomical structures of the central nervous system, including the spinal cord. However, later it became clear that a considerable overlap in relaxation times values can exist between normal and pathological states of the central nervous system, thus often making an accurate separation as well as characterisation of a lesion impossible (Perovitch, 1986 I; Perovitch, 1986 II). It has become apparent that certain substances may be necessary to enhance the image of certain features. Iodinated contrast media used in other neuroradiological procedures and magnetic resonance image enhancers have, obviously, the same purpose but vastly different properties.

At present, the most promising of these enhancers is Gadolinium Diethylen Triamine Penta-Acetic Acid (DTPA). Gadolinium-DTPA ${ }^{-}$appears to be especially suitable for clinical studies because of its strong effect on proton relaxation, its stability, rapid renal clearance, and good tolerance by patients. The injection of Gadolinium, when necessary, has in most instances improved the diagnostic quality of MRI of the brain. However, with regard to the spinal cord the experience with the intravenous injection of Gadolinium is so very 
Table IIIA The present major limitations of MRI of the spinal cord

1. Relative large slice thickness;

2. Long imaging time;

3. The gray-white matter distinction is seldom is seldom possible below the brain stem;

4. Significant degradation of image quality in scoliosis.

\section{Table IIIB}

5. Characterisation of a detected lesion is often not possible;

6. MRI cannot yet replace in all cases metrizamide or gas-myelography, nor the spinal cord angiography;

7. Paramagnetic enhancers may be needed to outline distinctly a lesion;

8. The filum terminale and nerve fibres cannot be identified.

limited at present that it is not possible to reach an objective conclusion (Perovitch, 1986, I; Perovitch, 1984).

It should be further mentioned that in addition to the proton MR images of the spinal cord our research in the past 2 years has been focussed on the possibility to obtain images of the distribution of other nuclei that may have greater diagnostic value than Hydrogen, such as Sodium $\left({ }^{23} \mathrm{Na}\right)$, Phosphorus $\left({ }^{31} \mathrm{P}\right)$, and Carbon $\left({ }^{13} \mathrm{C}\right)$. Hence in the future we may have the possibility to explore, under clinical conditions, the biomedical processes that occur in the spinal cord under normal and pathological circumstances (Perovitch, 1986, II).

\section{Conclusion}

In conclusion let us say that despite the current limitations, enumerated in Table III, A, and B, which are expected to be eliminated, MRI shows specific diagnostic potentials which add a new dimension to the investigation of spinal cord injury. The overall present clinical experience supports the view that MRI has much to offer, either as an adjunct to other diagnostic techniques, or as a primary investigative modality per se. The eventual introduction of paramagnetic enhancers such as Gadolinium may upgrade the diagnostic quality of MRI of spinal cord lesions. Thus, it could become easier to separate a posttraumatic intramedullary cyst from a cystic astrocytoma. However, further improvements in fundamental MR technology or image processing could minimise the clinical significance of paramagnetic enhancers. Namely, pulsing sequences alone may be able to do much of what is expected from paramagnetic enhancers (Perovitch, 1986, I).

\section{References}

Han JS, Benson JE, Young YS 1984 Magnetic resonance imaging in the spinal column and craniovertebral junction. In: Symposium on Magnetic Resonance Imaging. Alfidi RJ, Haage JR (eds). The Radiologic Clinics of North America 22:805-827.

Paushter DM, Modic MT 1984 Magnetic resonance imaging of the spine. Applied Radiology 13:61-68. 
Perovitch M 1986 (I) Application of 'contrast agents' in MR imaging. In: Proceedings of the International Congress of Clinical Neuroimaging. Wiener Medizinische Akademie Wien pp 44-46.

Perovitch M 1986 (II) Potential application of magnetic resonance spectroscopy in the clinical setting. In: Second Colloquium in Biological Sciences, Burrel CD, Strand FL (eds). Annals of the New York Academy of Sciences New York 463:337-338.

Perovitch M 1985 Neuroradiological evaluation of headache. In: Updating in Headache, Pfaffenrath V, Lundberg P-O, Sjaanstad O (eds). Springer Berlin Heidelberg New York pp 76-78.

Perovitch M 1984 Potential application of MR in brain disorders. In: Proceedings of the Annual Meeting of the American Association for the Advancement of Science. AAAS Publications Washington DC 84-4:177.

PEROVITCH M 1983 Neuroradiology of the sequelae of the spinal cord trauma. In: Trauma and Regeneration, Hume Adams J (ed). Acta Neurochirurgica Suppl. 32. Springer Wien New York pp 91-94.

PEROVITCH M 1981 Injuries of the spinal cord and meninges. In: Radiological Evaluation of the Spinal Cord, Vol. 1, Perovitch M (ed). CRC Press Boca Ra Florida pp 143-169. 